\title{
HÁBITOS DE LECTURA Y CONSUMO DE INFORMACIÓN EN ESTUDIANTES DE LA FACULTAD DE FILOSOFÍA Y LETRAS DE LA UNIVERSIDAD DE ZARAGOZA
}

\author{
José Antonio Salvador-Oliván* \\ Departamento de Ciencias de la Documentación e Historia de la Ciencia. Universidad de Zaragoza. \\ M. ${ }^{a}$ del Carmen Agustín-Lacruz ${ }^{* *}$ \\ Departamento de Ciencias de la Documentación e Historia de la Ciencia. Universidad de Zaragoza.
}

\begin{abstract}
Resumen: El objetivo del estudio es explorar los hábitos de lectura y consumo de información de estudiantes de la Facultad de Filosofía y Letras de la Universidad de Zaragoza. El estudio es descriptivo, transversal. Se utilizó un cuestionario autoadministrado para recoger los datos de 561 estudiantes. El análisis de datos incluyó estadísticos descriptivos y el test de chi cuadrado.

Los resultados revelan que los estudiantes universitarios son lectores frecuentes de una variedad amplia de tipos de información relacionada con el ocio, con el proceso de enseñanza-aprendizaje o simplemente periodística, siendo los dispositivos más empleados el ordenador y el smartphone, y los lugares más habituales de lectura el domicilio, la biblioteca y los espacios públicos. La política, economía y deportes son los temas más leídos por los hombres, mientras que los contenidos en revistas no académicas (salud, moda, sociedad, horóscopo), aunque son los menos leídos, son preferidos por las mujeres.
\end{abstract}

Palabra clave: Hábitos de lectura; consumo de información; estudiantes universitarios; preferencias de lectura; dispositivos de lectura; Facultad de Filosofía y Letras; España.

\begin{abstract}
Title: INFORMATION READING AND CONSUMPTION HABITS AMONG THE UNDERGRADUATE STUDENTS IN HUMANITIES FACULTY IN SARAGOSSA UNIVERSITY.

Abstract: Objective: This study was carried out to assess information consumption and reading habits among undergraduate students from Humanitities Faculty at Saragossa University.

Methods: A descriptive cross sectional approach was adopted. It involved 561 undergraduate students. A questionnaire for measuring reading habits was used for data collection. Frequency distribution percentage and cross tabulation with Chi square method were used in analyzing data.

Results: The findings revealed that respondents read a wide range and types of information related with leisure, the process of teaching-learning or informative, being the most devices the computer and the smartphone. The most habitual places of reading are the domicile, the library and the public spaces. Some differences in reading preferences were observed between male and female students; men read more topics of policy, economics and sports while women read more topics related with health, fashion, society and horoscope

Keywords: Reading habits; information consumption; undergraduate students; reading preferences; lecture devices; Humanities Faculty; Spain.
\end{abstract}

\section{INTRODUCCIÓN}

La lectura es un proceso de comunicación dinámico e interactivo que incluye conocimientos, habilidades y estrategias que las personas adquieren según sus experiencias y mediante la interacción con otros sujetos. No sólo es una capacidad de decodificación de signos escritos adquirida en las etapas iniciales del aprendizaje, sino un proceso intelectual y cognitivo complejo de naturaleza lingüística, en el que cada lector aporta su propia construcción de sentido, adquiriendo nuevo conocimiento a través de la comprensión, atribución de significación e interpretación del texto.

Puede decirse que la lectura está en la base de los aprendizajes que conforman la educación de un individuo. Por ello, la adquisición de la competencia lectora -entendida como capacidad de comprender, utilizar y analizar textos escritos para alcanzar los objetivos del sujeto lector, y desarrollar sus conocimientos y posibilidades y participar en la sociedad- proporciona a las personas, a lo largo de toda la vida, los recursos comunicativos y los instrumentos lingüísticos necesarios para hacer frente a las exigencias de las complejas sociedades modernas, con sus extensos aparatos burocráticos, sus instituciones formales y sus complejos sistemas sociales, educativos y jurídicos.

\footnotetext{
*jaso@unizar.es

***agustin@unizar.es
}

Recibido: 07-07-2014; 2ª versión: 23-12-2014; aceptado: 09-01-2015.

SALVADOR OLIVÁN, J.A. y AGUSTÍN LACRUZ, M.C. Hábitos de lectura y consumo de información en estudiantes de la Facultad de Filosofía y Letras de la Universidad de Zaragoza. Anales de Documentación, 2015, vol. 18, n 1 . Disponible en: http://dx.doi.org/10.6018/analesdoc.18.1.201971 
JOSÉ ANTONIO SALVADOR Y MARÍA DEL CARMEN AGUSTÍN

El desarrollo del hábito lector se considera un recurso importante para la formación de la personalidad y la mejora de las capacidades cognitivas de los individuos (Ögeyik y Akyay, 2009) al enriquecer su intelecto, proporcionarles una mejor comprensión de los problemas e influir en sus actitudes y comportamientos (Quadri y Abomoge, 2013).

La lectura adquiere gran importancia en el ámbito de la educación en todas las etapas formativas y especialmente en la universitaria como herramienta para generar y comunicar conocimiento y también como portadora de una actitud vital que implica mantener la mente abierta, alerta y libre (Rodríguez Legido, 2009). Sin embargo, los resultados que obtienen los estudiantes españoles al evaluar su comprensión lectora digital han empeorado en la última etapa, habiendo obtenido 466 puntos (la media de la OCDE ha sido de 497), frente a los 475 de 2009 (Ministerio de Educación, Cultura y Deporte, 2013).

Tradicionalmente, los objetos de la lectura han sido textos escritos en soporte papel, pero el crecimiento de internet y el desarrollo y uso generalizado de las nuevas tecnologías junto con la aparición de nuevos dispositivos móviles, han supuesto un cambio en el panorama de la distribución y acceso a la información y, en consecuencia, en su lectura. Por ello, Zoido, analista de la OCDE, señala que "el tipo de competencias que va a hacer falta para el día de mañana se acerca cada vez más a la lectura en soporte digital que en papel” (García de Blas, 2014).

El campo de la educación no ha sido ajeno a estos cambios y son muchos los estudios que se han realizado, tanto en las escuelas y en población infantil -orientados al comportamiento lector (Yubero y Larrañaga, 2010) y a su relación con el aprendizaje (Gil Flores, 2011) y la comprensión lectora (Schugar, Schugar y Penny, 2011)- como en estudiantes universitarios (Thanuskodi, 2011; Quadri y Abomoge, 2013), donde los trabajos han estado dirigidos a evaluar el impacto de las tecnologías informáticas en los hábitos de lectura (Shen, 2006), a conocer las preferencias de los postgraduados (Noorizad, 2011), determinar si los estudiantes universitarios leen en papel o en pantalla (Keller, 2012) o más concretamente libros impresos o electrónicos (Cumaolgu, Sacici y Torum, 2013), así como analizar la usabilidad de los lectores de libros electrónicos (Olsen, Kleivset y Langset, 2013) o los efectos de los libros electrónicos sobre el aprendizaje (Szapkiw et al., 2013). En la revisión realizada por Cull (2011) se examinó el impacto social, tecnológico, conductual y neurocientífico que ha tenido internet en la práctica de la lectura desde diferentes disciplinas. Recientemente se ha estudiado también la relación que existe entre los modos de interacción social surgidos con la aparición de las tecnologías digitales y la disminución del tiempo dedicado a la lectura por los jóvenes (Akanda, Hok y Hasan, 2013).

En España, los primeros estudios sobre hábitos lectores datan de 1964, promovidos por empresas editoriales que se interesaban por conocer el perfil de los lectores, considerados como potenciales compradores. A lo largo de los años 70 y 80, el Instituto de Opinión Pública, la Asociación Nacional de Archiveros, Bibliotecarios y Arqueólogos, el Instituto Nacional de Estadística y el Ministerio de Cultura, entre otras instituciones, realizaron encuestas a nivel nacional para conocer el número de lectores, sus frecuencias y preferencias de lectura. En 1988, el Instituto de la Juventud, dependiente del Ministerio de Asuntos Sociales, estudió los hábitos de la población juvenil española, incluyendo apartados concretos sobre los hábitos lectores. La década de los 90 impulsó análisis más específicos orientados hacia los equipamientos, consumos y hábitos culturales, incluyendo estudios segmentados para conocer las preferencias de consumo y estrategias de venta. Estos abordajes abrieron camino a iniciativas como la realizada anualmente desde el año 2000 por la Federación del Gremio de Editores para conocer, entre otros aspectos, hábitos y comportamientos lectores, tipología y frecuencias de lectura (Larrañaga Rubio, 2004).

A lo largo de este tiempo, ha crecido la bibliografía sobre las conductas lectoras de niños o escolares si bien no se han incrementado, en la misma medida, los estudios sobre hábitos de lectura en universitarios.

En 1996 se publicó el primer trabajo específico sobre hábitos lectores de estudiantes universitarios españoles para describir las lecturas académicas de estudiantes de Psicología y Magisterio de la Universidad de Barcelona (Teberosky, Guardia y Escoriza, 1996). Años más tarde, en 2003 Díaz Gómez y Gámez Armas se ocuparon de analizar las relaciones entre factores motivacionales y los hábitos de lectura de un grupo de estudiantes de Pedagogía y Psicología de la Universidad de La Laguna. En 2004, Larrañaga Rubio, también desde una perspectiva psicológica, defendió una tesis doctoral sobre el proceso de educación de los hábitos lectores de los estudiantes de la Universidad de Castilla-La Mancha, en la que se estudiaban las variables psicosociales que intervienen en el desarrollo de la lectura como comportamiento de ocio y se analizaba la influencia del currículo universitario en la conducta lectora. El estudio se realizó encuestando a estudiantes de diversos estudios y niveles, de cinco universidades (Castilla-La Mancha, Complutense de Madrid, Alcalá de Henares, León y Alicante). Esta línea de trabajo fue proseguida por la autora (Larrañaga y Martínez, 2004) y ampliada junto a sus dos directores de tesis (Larrañaga, Yubero y Cerrillo, 2008; Yubero, Larrañaga y Cerrillo, 2009) estudiando universitarios españoles pertenecientes a doce universidades públicas españolas. Los resultados obtenidos confirmaron una frecuencia superior en la conducta lectora de los estudiantes en 
general y de los universitarios en particular, con respecto al resto de la población. Del mismo modo, se encontraron porcentajes superiores de lectura en las mujeres universitarias que en los varones.

Este panorama general fue completado en 2009 con un estudio comparativo de hábitos de estudiantes, profesorado y personal administrativo de las universidades andaluzas (Rodriguez Legido, 2009) y más tarde, por dos estudios sobre textos específicos (Santos Díez y Pérez Dasilva, 2010; García Delgado, 2011). El primero de estos trabajos estudió la percepción de estudiantes de Periodismo de la Universidad del País Vasco de las ediciones impresas de los diarios gratuitos de información general, considerando aspectos como su calidad, hábitos de consumo, cabeceras preferidas y secciones, así como su complementariedad con los medios tradicionales (prensa, radio, televisión e internet). Por su parte, García Delgado se ocupó de la lectura recreativa de la Universidad Europea de Madrid, tratando de cuantificar el tiempo dedicado a la lectura en comparación con otras actividades de consumo de información (televisión, radio).

Desde un punto de vista temático, los estudios sobre hábitos de lectura en estudiantes universitarios españoles se han centrado en cuestiones diversas, como la tipología de las lecturas académicas (Teberosky, Guardia y Escoriza, 1996); los aspectos motivacionales (Díaz Gómez y Gámez Armas, 2003); la influencia de variables psico-sociales (Larrañaga Rubio, 2004; Larrañaga y Martínez, 2004; Larrañaga; Yubero y Cerrillo, 2008; Yubero; Larrañaga y Cerrillo, 2009); las diferencias de los diferentes grupos académicos (Rodríguez Legido, 2009); la lectura de prensa gratuita (Santos Díez y Pérez Dasilva, 2010); la lectura recreativa (García Delgado, 2011) y recientemente, libros digitales y su comparación con otros países (Alonso Arévalo; Cordón García y Gómez Díaz, 2014).

En la actualidad, los estudiantes universitarios españoles pertenecen a una generación integrada por los denominados “nativos digitales” (Prensky, 2001), nacidos después de la implantación de las tecnologías de la información, que han crecido rodeados de dispositivos y herramientas propias de la era digital, que utilizan de manera habitual para comunicarse, formarse y entretenerse.

Para ellos, la lectura no es un acto unívoco. Leen muchos textos diferentes de muchas maneras diferentes y por muy diferentes razones, sea en papel o en texto digital, sean periódicos, revistas, manuales, libros de texto, literatura, etc. (Brown, 2001). En la actualidad, la tecnología informática está integrada en casi todos los aspectos del aprendizaje en la educación universitaria y nos encontramos que cada vez es más frecuente simultanear la lectura de textos de correos electrónicos, páginas web, mensajes de teléfonos móviles, artículos de revistas digitales y libros electrónicos.

\section{OBJETIVOS}

En un contexto de cambio estructural continuo, determinado por factores como la disponibilidad de un amplio catálogo documental digital, la coexistencia simultánea de la edición analógica y digital, la extensión de los dispositivos electrónicos de lectura, tanto en versión de tinta electrónica -ereaders-, como en tabletas, y el crecimiento de la oferta proporcionada por las grandes librerías online internacionales en nuestro país, resulta de interés identificar la extensión y las características de las nuevas prácticas lectoras y las diferentes manifestaciones que el polimorfismo y la multimodalidad de la lectura digital adopta entre los estudiantes universitarios, abordando sus hábitos y frecuencias de lectura, usos y consumos, tipologías documentales así como las características y funcionalidades de los soportes utilizados.

El planteamiento de este estudio consiste, por tanto, en conocer si los recursos electrónicos han sustituido en gran medida a los recursos impresos y los hábitos de lectura de los diferentes tipos de información presentes en la actualidad a través de diferentes medios. Por ello, el objetivo general consiste en explorar los hábitos de lectura y consumo de información y los medios más utilizados por la población universitaria en una facultad de humanidades. Concretamente, se desea conocer:

- tipo de información y dispositivos más utilizados en cada tipo

- frecuencia con que se lee la información

- temáticas de más interés y diferencias en cuanto a sexo

- lugares más habituales de lectura

- preferencias de lectura en soporte impreso o digital

\section{MÉTODO}

Población de estudio: La población está formada por todos los estudiantes universitarios matriculados en los grados y licenciaturas de la Facultad de Filosofía y Letras de la Universidad de Zaragoza en el curso 2012-2013. El número total de estudiantes es de 2733. 
Tipo de estudio: Descriptivo, transversal.

Diseño del estudio: Muestreo por conglomerados monoetápico estratificado por titulación.

Se ha definido como conglomerado cada uno de los grupos administrativos de la titulación en la que se matricula el estudiante. Existen 10 titulaciones, 3 de ellas con el grado completo, y 7 de ellas con los tres primeros cursos de grado y los dos últimos de licenciatura. De ellas, tres titulaciones tienen dos grupos, el resto solo tienen 1 grupo, con lo que el número total de conglomerados es:

3 grados completos $\mathrm{x} 4$ cursos $\mathrm{x} 1$ grupo $=12$ conglomerados

4 grados incompletos $\mathrm{x} 3$ cursos $\mathrm{x} 1$ grupo $=12$ conglomerados

3 grados incompletos $\times 3$ cursos $x 2$ grupos $=18$ conglomerados

4 licenciaturas $\mathrm{x} 2$ cursos $\mathrm{x} 1$ grupo $=8$ conglomerados

3 licenciaturas $\mathrm{x} 2$ cursos $\mathrm{x} 2$ grupos $=12$ conglomerados

Total: 62 conglomerados.

El número de alumnos por conglomerado es desigual. Para que estuvieran representados los alumnos de todas las titulaciones, se optó por seleccionar al azar dos conglomerados de cursos diferentes por titulación.

Tamaño muestral: Para un tamaño poblacional de 2733 estudiantes, un error alfa del 5\%, una proporción esperada del 0,5, y una precisión del 4\%, el tamaño muestral inicial fue de 493 alumnos.

Una vez seleccionados 2 conglomerados de cada una de las 10 titulaciones, el tamaño de la muestra final obtenido ha sido de 561. La distribución de los alumnos por titulación se muestra en la Tabla I, donde se puede apreciar que algunos estudios están más representados en la población y otros menos representados; esto se debe a que los cuestionarios se pasaron en clase y no todos los alumnos matriculados asisten.

\begin{tabular}{|l|r|r|r|r|}
\hline & \multicolumn{2}{|c|}{ Muestra final } & \multicolumn{2}{c|}{ Población } \\
\hline Estudios Clásicos & 28 & $5,0 \%$ & 92 & $3,4 \%$ \\
\hline Estudios Ingleses & 63 & $11,2 \%$ & 476 & $17,4 \%$ \\
\hline Filología Hispánica & 74 & $13,2 \%$ & 272 & $10,0 \%$ \\
\hline Filosofía & 38 & $6,8 \%$ & 153 & $5,6 \%$ \\
\hline Geografía & 45 & $8,0 \%$ & 185 & $6,8 \%$ \\
\hline Historia & 112 & $20,0 \%$ & 661 & $24,2 \%$ \\
\hline Historia del Arte & 60 & $10,7 \%$ & 408 & $14,9 \%$ \\
\hline Información y Documentación & 23 & $4,1 \%$ & 95 & $3,5 \%$ \\
\hline Lenguas Modernas & 36 & $6,4 \%$ & 143 & $5,2 \%$ \\
\hline Periodismo & 82 & $14,6 \%$ & 248 & $9,1 \%$ \\
\hline Total & 561 & $100,0 \%$ & 2733 & $100,0 \%$ \\
\hline
\end{tabular}

Tabla I. Distribución de la muestra final y población de estudio por titulación.

Posteriormente, una vez definido el tamaño final de la muestra, se recalculó el error de muestreo, siendo la precisión de 3,7\%.

Cuestionario utilizado: Se diseñó un cuestionario estructurado (Anexo 1) con preguntas sobre los hábitos y tipos de lectura relacionados con el uso de diferentes dispositivos (tanto en papel como electrónicos) agrupadas en 5 bloques correspondientes a cada uno de los objetivos específicos del estudio.

Forma de administración: Encuesta autoadministrada. Una persona se encargó de acudir a las clases de los grupos seleccionados, explicar los objetivos y la importancia de la investigación, repartir los cuestionarios y recogerlos una vez cumplimentados. La encuesta se llevó a cabo en los meses de marzo a mayo de 2013.

Tipología de lectores: Se sigue la tipología utilizada en los informes de hábitos de lectura y compra de libros realizados por la Federación de Gremios de Editores de España (2013). Se clasifica a los lectores según la frecuencia; en lectores frecuentes (aquellos que leen por lo menos alguna vez a la semana), lectores habituales (aquellos que leen al menos una vez al mes), lectores ocasionales (con frecuencia menor que semanal y de alguna vez al mes o al trimestre) y no lectores (que no leen casi nunca o nunca). 
Proceso y análisis de datos: Los datos se introdujeron y procesaron con el programa SPSS v. 19, obteniendo los estadísticos descriptivos y aplicando el test de chi cuadrado para analizar las diferencias entre grupos.

\section{RESULTADOS}

El número de estudiantes encuestados ha sido de 561, con una edad media de 21,6 años, de los que 224 son hombres (39,9\%) con una edad media de 22,1 años y 337 mujeres (60,1\%) con una edad media de 21,3 años.

Tipos de información y formatos utilizados: La mayoría de los estudiantes universitarios son lectores frecuentes de libros de texto o material docente y literatura, más en papel que en formato digital, debido quizá en el caso de los libros de texto a que solo se encuentran en formato impreso aunque se compensa porque material docente preparado por el profesorado solo está disponible en formato digital en las plataformas docentes. Los valores de lectura de prensa y revistas son más bajos y no llega a los dos tercios los estudiantes que declaran leer con frecuencia este tipo de información, que en el caso de la prensa es muy similar tanto en formato papel como digital, aunque en el caso de las revistas apenas 1 de cada 4 estudiantes utilizan el formato digital.

El hábito de lectura más destacado en formato digital es el consumo de páginas web (98,2\%) seguido de la consulta de correo electrónico y SMS (96,6\%) y de la escucha de música y audio (95,5\%). La lectura y uso de blogs, foros, chats y WhatsApps (un 94,3\%), así como la descarga o visionado de películas y vídeos (92,2\%) y la interacción en las redes sociales $(88,6 \%)$ es también muy habitual y destacada por parte de los estudiantes.

\begin{tabular}{|l|cc|cc|}
\hline & \multicolumn{2}{|c}{ Papel } & \multicolumn{2}{c|}{ Digital } \\
\hline Prensa & 348 & $62,0 \%$ & 361 & $64,3 \%$ \\
\hline Revistas & 360 & $64,2 \%$ & 133 & $23,7 \%$ \\
\hline Libros de texto/material docente & 509 & $90,7 \%$ & 311 & $55,4 \%$ \\
\hline Literatura & 494 & $88,1 \%$ & 181 & $32,3 \%$ \\
\hline Páginas web & & & 551 & $98,2 \%$ \\
\hline Correo electrónico/SMS & & 542 & $96,6 \%$ \\
\hline Blogs, foros, chats, WhatsApps & & 529 & $94,3 \%$ \\
\hline Redes sociales & & 497 & $88,6 \%$ \\
\hline Películas, vídeos & & 517 & $92,2 \%$ \\
\hline Música, audio & & 536 & $95,5 \%$ \\
\hline
\end{tabular}

Tabla II. Tipos de información leída y formatos utilizados al menos una vez por semana.

Dispositivos utilizados en la lectura digital: El 10\% de los estudiantes utiliza solo un dispositivo para la lectura de información digital, el 35,3\% utiliza dos dispositivos, el 38\% tres dispositivos, el 12,1\% cuatro dispositivos y solo el 4,1\% utiliza cinco dispositivos diferentes.

Los dispositivos más utilizados son el ordenador portátil (90,4\%) seguido del smartphone (76,5\%), lo que indica que la mayoría de los estudiantes disponen de ordenador portátil.

El ordenador portátil es el dispositivo más utilizado para la lectura de todos los tipos de información analizados, siendo sus usos más habituales el acceso a páginas web (96,1\%), la lectura de correo electrónico y SMS (90,9\%), así como la descarga de música y audio (90,2\%), visionado o descarga de películas y vídeos (89,2\%) y las redes sociales (86,8\%). Los usos más habituales del ordenador de sobremesa son los mismos que los del ordenador portátil.

El smartphone es utilizado por algo más de las tres cuartas partes de los estudiantes, siendo el segundo dispositivo más utilizado, principalmente para la comunicación a través de blogs, foros, chats o WhatsApps, redes sociales y correo electrónico o SMS.

La tableta es un dispositivo poco presente entre la población universitaria, solo lo utiliza el 15\% de los estudiantes, siendo su uso más habitual para la navegación por páginas web, redes sociales, correo electrónico, y blogs, foros o WhatsApps, coincidiendo también, aunque en menor grado, con los usos de los ordenadores.

El uso del lector de libros electrónicos está un poco más extendido que el de la tableta, con un 18,7\% de los estudiantes que disponen de este dispositivo, siendo utilizado preferentemente para el principal objetivo con que fue creado, la lectura de literatura (54,3\%), así como la lectura de libros de texto y material docente (46,7\%). 


\begin{tabular}{|c|c|c|c|c|c|c|c|c|c|c|c|}
\hline \multirow[b]{2}{*}{ Prensa } & \multicolumn{2}{|c|}{$\begin{array}{l}\text { Ordenador } \\
\text { sobremesa }\end{array}$} & \multicolumn{2}{|c|}{$\begin{array}{l}\text { Ordenador } \\
\text { portátil }\end{array}$} & \multicolumn{2}{|c|}{ Smartphone } & \multicolumn{2}{|c|}{ Tableta } & & \multirow{2}{*}{$\begin{array}{c}\text { TOTAL } \\
361\end{array}$} \\
\hline & 173 & $49,0 \%$ & 300 & $59,2 \%$ & 210 & $49,0 \%$ & 45 & $53,6 \%$ & 19 & $18,1 \%$ & \\
\hline Revistas & 67 & $19,0 \%$ & 108 & $21,3 \%$ & 56 & $13,1 \%$ & 11 & $13,1 \%$ & 8 & $7,6 \%$ & 133 \\
\hline $\begin{array}{l}\text { Libros de texto/mat } \\
\text { docente }\end{array}$ & 162 & $45,9 \%$ & 260 & $51,3 \%$ & 57 & $13,3 \%$ & 30 & $35,7 \%$ & 49 & $46,7 \%$ & 311 \\
\hline Literatura & 84 & $23,8 \%$ & 140 & $27,6 \%$ & 49 & $11,4 \%$ & 22 & $26,2 \%$ & 57 & $54,3 \%$ & 181 \\
\hline Páginas web & 322 & $90,7 \%$ & 488 & $96,1 \%$ & 359 & $83,5 \%$ & 64 & $72,7 \%$ & 24 & $19,0 \%$ & 551 \\
\hline $\begin{array}{l}\text { Correo } \\
\text { electrónico/SMS }\end{array}$ & 313 & $88,2 \%$ & 462 & $90,9 \%$ & 375 & $87,2 \%$ & 58 & $65,9 \%$ & 8 & $6,3 \%$ & 542 \\
\hline $\begin{array}{l}\text { Blogs, foros, chats, } \\
\text { WhatsApps }\end{array}$ & 269 & $75,8 \%$ & 422 & $83,1 \%$ & 397 & $92,3 \%$ & 56 & $63,6 \%$ & 11 & $8,7 \%$ & 529 \\
\hline Redes sociales & 276 & $78,2 \%$ & 440 & $86,8 \%$ & 374 & $87,2 \%$ & 60 & $71,4 \%$ & 6 & $5,7 \%$ & 497 \\
\hline Películas, vídeos & 282 & $79,4 \%$ & 453 & $89,2 \%$ & 219 & $50,9 \%$ & 52 & $59,1 \%$ & 10 & $7,9 \%$ & 517 \\
\hline Música, audio & 297 & $83,7 \%$ & 458 & $90,2 \%$ & 358 & $83,3 \%$ & 49 & $55,7 \%$ & 20 & $15,9 \%$ & 536 \\
\hline TOTAL & 353 & $62,9 \%$ & 507 & $90,4 \%$ & 429 & $76,5 \%$ & 84 & $15,0 \%$ & 105 & $18,7 \%$ & 561 \\
\hline
\end{tabular}

Tabla III. Dispositivos utilizados en el consumo y lectura de información digital.

Todos los estudiantes salvo 4 utilizan el ordenador (99,3\%), ya sea portátil o de sobremesa. En la Tabla IV se presenta su uso para los distintos tipos de información, siendo el más habitual la consulta de páginas web (97,8\%), seguido del correo electrónico (94\%), descarga de música o audio (93,9\%) y visionado de películas o vídeos (91,9\%). El uso menos frecuente es la lectura de revistas (22,2\%). Al analizar el uso del ordenador para cada tipo de información destaca que los porcentajes son muy altos: en todos los casos supera el $90 \%$ salvo en literatura que es un poco inferior (88,4\%), siendo el dispositivo más utilizado para la lectura de todo tipo de información.

\begin{tabular}{|c|c|c|c|}
\hline & $\mathbf{N}^{\mathbf{0}}$ & $\%^{a}$ & $\%^{b}$ \\
\hline Prensa & 348 & $62,48 \%$ & $96,40 \%$ \\
\hline Revistas & 124 & $22,26 \%$ & $93,23 \%$ \\
\hline Libros de texto/material docente & 296 & $53,14 \%$ & $95,18 \%$ \\
\hline Literatura & 160 & $28,73 \%$ & $88,40 \%$ \\
\hline Páginas web & 545 & $97,85 \%$ & $98,91 \%$ \\
\hline Correo electrónico/SMS & 524 & $94,08 \%$ & $96,68 \%$ \\
\hline Blogs, foros, chats, WhatsApps & 479 & $86,00 \%$ & $90,55 \%$ \\
\hline Redes sociales & 490 & $87,97 \%$ & $98,59 \%$ \\
\hline Películas, vídeos & 512 & $91,92 \%$ & $99,03 \%$ \\
\hline Música, audio & 523 & $93,90 \%$ & $97,57 \%$ \\
\hline TOTAL & 557 & $100 \%$ & \\
\hline
\end{tabular}

Tabla IV. Uso de ordenador (sobremesa o portátil) en la lectura y consumo de información en formato digital.

${ }^{\text {a }}$ Base porcentaje: número total de estudiantes con ordenador.

${ }^{\mathrm{b}}$ Base porcentaje: número total de estudiantes que leen el tipo de información.

Fuentes de información utilizadas en internet y canales de comunicación: Respecto a la frecuencia de uso de los canales de comunicación, destaca que las redes sociales son utilizadas todos o casi todos los días por el 79,7\% de los estudiantes, porcentaje que sube hasta el 88,6\% cuando el uso es semanal; igual ocurre con el correo electrónico, un servicio de comunicación utilizado por el 77,4\% de los estudiantes de manera diaria y que asciende hasta el $94,2 \%$ cuando su uso es semanal. La mitad de los estudiantes utiliza diariamente los blogs o chats, y casi el 70\% lo hace semanalmente.

En cuanto a las fuentes de información, la prensa es leída por algo menos de la mitad de los estudiantes de forma diaria, si bien 2 de cada 3 la leen una vez por semana. Las revistas son la fuente de información leída con menos asiduidad, con un 43,5\% de los estudiantes que nunca o casi nunca leen información de revistas académicas o científicas y un 44,5\% que nunca o casi nunca leen revistas de ocio. 


\begin{tabular}{|l|c|c|c|c|c|c|} 
& Nunca & $\begin{array}{c}\text { Casi } \\
\text { nunca }\end{array}$ & $\begin{array}{c}\text { Al menos } \\
\text { una vez cada } \\
\text { tres meses }\end{array}$ & $\begin{array}{c}\text { Al menos } \\
\text { una vez al } \\
\text { mes }\end{array}$ & $\begin{array}{c}\text { Una o dos } \\
\text { veces por } \\
\text { semana }\end{array}$ & $\begin{array}{c}\text { Todos o } \\
\text { casi todos } \\
\text { los días }\end{array}$ \\
\hline Revistas de ocio & $18,7 \%$ & $25,8 \%$ & $11,4 \%$ & $19,4 \%$ & $11,2 \%$ & $6,4 \%$ \\
\hline $\begin{array}{l}\text { Revistas académicas, } \\
\text { científicas }\end{array}$ & $16,0 \%$ & $27,5 \%$ & $14,8 \%$ & $24,8 \%$ & $9,3 \%$ & $2,5 \%$ \\
\hline Prensa & $3,7 \%$ & $8,0 \%$ & $6,4 \%$ & $13,0 \%$ & $20,3 \%$ & $44,0 \%$ \\
\hline $\begin{array}{l}\text { Libros de texto/material } \\
\text { docente }\end{array}$ & $2,1 \%$ & $5,5 \%$ & $5,0 \%$ & $19,6 \%$ & $31,9 \%$ & $23,5 \%$ \\
\hline Literatura & $9,8 \%$ & $15,5 \%$ & $10,2 \%$ & $21,7 \%$ & $18,0 \%$ & $14,3 \%$ \\
\hline Redes sociales & $3,6 \%$ & $2,7 \%$ & $1,4 \%$ & $1,6 \%$ & $8,9 \%$ & $79,7 \%$ \\
\hline Correo electrónico & $0,5 \%$ & $0,9 \%$ & $0,7 \%$ & $2,9 \%$ & $16,8 \%$ & $77,4 \%$ \\
\hline Blogs/foros/chats & $4,8 \%$ & $8,4 \%$ & $5,9 \%$ & $10,7 \%$ & $19,6 \%$ & $49,7 \%$ \\
\hline
\end{tabular}

Tabla V. Frecuencia de uso de diversas fuentes de información en internet y canales de comunicación.

Temas de lectura en internet: Las temáticas que más interesan a los universitarios encuestados y que consumen todos o casi todos los días, son la música (41,3\%), política (34,2\%), cine (29,4\%) deportes (27,4\%), meteorología $(21,6 \%)$ y economía (21,4\%), con porcentajes de consumo semanal en estos temas que oscilan entre el $69,4 \%$ de la música hasta el 42,9\% de los deportes. Cabe destacar, por tanto, que la política ocupa un lugar preferente entre los temas más leídos por este segmento de público.

Los temas que menos interés despiertan son el horóscopo (un 58,5\% no lo lee nunca y un 19,4\% casi nunca), los relacionados con la sociedad y crónica rosa (37,0\% nunca y 23,9\% casi nunca) y la alimentación y dieta (22,7\% nunca y 26,7\% casi nunca).

\begin{tabular}{|l|c|c|c|c|c|c|} 
& Nunca & $\begin{array}{c}\text { Casi } \\
\text { nunca }\end{array}$ & $\begin{array}{c}\text { Al menos } \\
\text { una vez cada } \\
\text { tres meses }\end{array}$ & $\begin{array}{c}\text { Al menos } \\
\text { una vez al } \\
\text { mes }\end{array}$ & $\begin{array}{c}\text { Una o dos } \\
\text { veces por } \\
\text { semana }\end{array}$ & $\begin{array}{c}\text { Todos o } \\
\text { casi todos } \\
\text { los días }\end{array}$ \\
\hline Educación & $4,2 \%$ & $13,1 \%$ & $10,6 \%$ & $24,5 \%$ & $31,6 \%$ & $16,1 \%$ \\
\hline Política & $9,2 \%$ & $11,6 \%$ & $6,9 \%$ & $11,6 \%$ & $26,6 \%$ & $34,2 \%$ \\
\hline Economía & $10,1 \%$ & $15,9 \%$ & $11,2 \%$ & $15,5 \%$ & $26,0 \%$ & $21,4 \%$ \\
\hline Salud & $6,0 \%$ & $19,7 \%$ & $16,6 \%$ & $29,9 \%$ & $20,0 \%$ & $7,8 \%$ \\
\hline $\begin{array}{l}\text { Medio } \\
\text { ambiente/naturaleza }\end{array}$ & $7,3 \%$ & $17,7 \%$ & $19,9 \%$ & $26,9 \%$ & $19,9 \%$ & $8,2 \%$ \\
\hline Consumo & $12,1 \%$ & $25,0 \%$ & $17,0 \%$ & $24,6 \%$ & $16,7 \%$ & $4,5 \%$ \\
\hline Deportes & $20,1 \%$ & $18,8 \%$ & $8,2 \%$ & $10,0 \%$ & $15,5 \%$ & $27,4 \%$ \\
\hline Sociedad/crónica rosa & $37,0 \%$ & $23,9 \%$ & $9,8 \%$ & $9,8 \%$ & $13,5 \%$ & $6,0 \%$ \\
\hline Alimentación/dieta & $22,7 \%$ & $26,7 \%$ & $17,6 \%$ & $17,2 \%$ & $11,6 \%$ & $4,2 \%$ \\
\hline Empleo & $13,6 \%$ & $17,5 \%$ & $16,7 \%$ & $18,7 \%$ & $19,3 \%$ & $14,2 \%$ \\
\hline Moda & $25,4 \%$ & $17,8 \%$ & $12,3 \%$ & $18,1 \%$ & $13,9 \%$ & $12,5 \%$ \\
\hline Música & $2,5 \%$ & $4,5 \%$ & $7,4 \%$ & $16,1 \%$ & $28,1 \%$ & $41,3 \%$ \\
\hline Cine & $2,0 \%$ & $6,0 \%$ & $9,3 \%$ & $20,8 \%$ & $32,5 \%$ & $29,4 \%$ \\
\hline Meteorología & $8,3 \%$ & $15,4 \%$ & $8,5 \%$ & $20,5 \%$ & $25,7 \%$ & $21,6 \%$ \\
\hline Horóscopo & $58,5 \%$ & $19,4 \%$ & $6,3 \%$ & $6,7 \%$ & $4,3 \%$ & $4,7 \%$ \\
\hline Humor: chistes, cómics & $17,0 \%$ & $18,2 \%$ & $12,6 \%$ & $15,5 \%$ & $18,1 \%$ & $18,6 \%$ \\
\hline
\end{tabular}

Tabla VI. Frecuencia de lectura sobre diversos temas de información en internet.

Algunos temas tienen una representación muy equilibrada en todas las horquillas temporales, por lo que se observan consumos de diversa periodicidad, tal es el caso del empleo, moda, consumo o humor. En este sentido, cabe subrayar que el empleo no es un tema de interés preferente para todos los estudiantes, posiblemente dependa del curso en el que estén matriculados, más o menos próximos a finalizar los estudios universitarios.

El tema educativo sí presenta una lectura habitual y frecuente, en la mayoría de los casos una o dos veces a la semana (31,6\%), al menos una vez al mes (24,5\%) o, incluso, todos o casi todos los días (16,1\%). 
La temática de medio ambiente no es de las más leídas, pero el 26,9\% suele hacerlo al menos una vez al mes, el 19,9\% solo cada tres meses, y también el 19,9\% una o dos veces por semana. Con similar tendencia se comporta el tema de salud.

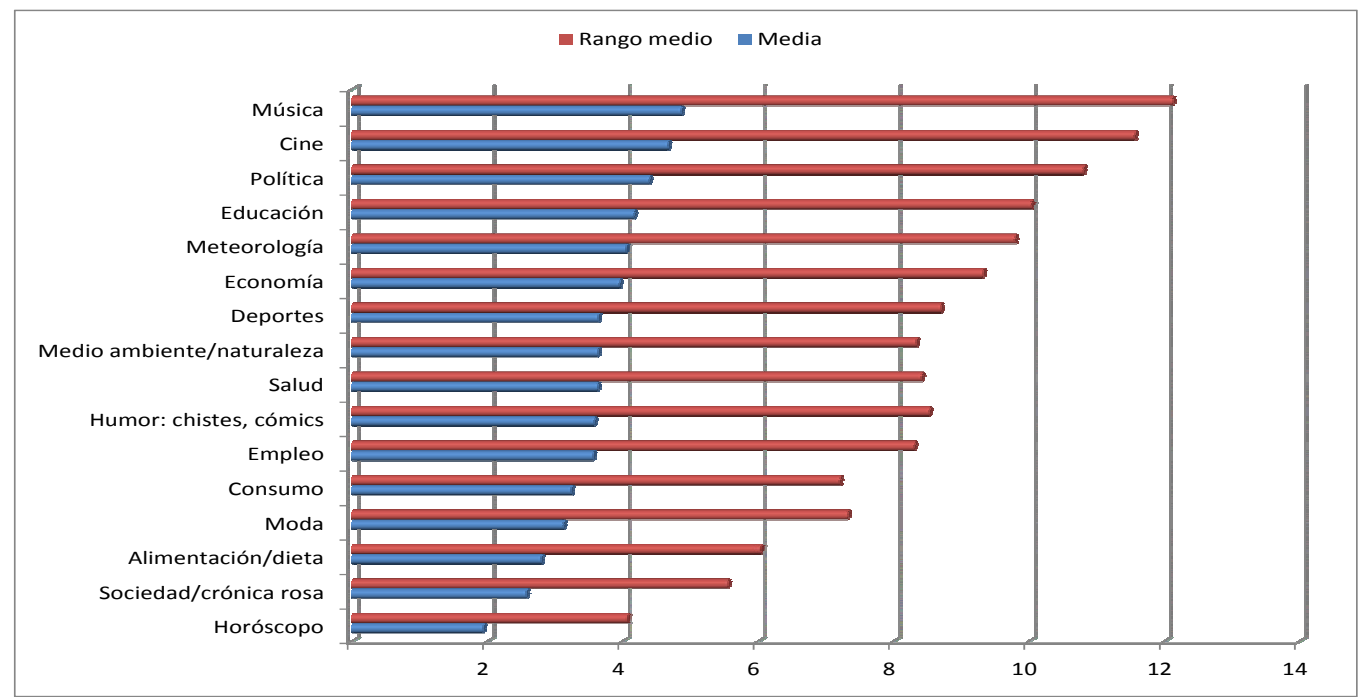

Figura 1. Temas de lectura más frecuentes ordenados por puntuación media y rango medio. Rangos medios obtenidos con el test de Friedman.

En la Figura 1 se presentan los temas de lectura ordenados según la frecuencia media de uso, observándose que es muy similar al orden según el rango medio obtenido. La música, el cine, la política y la educación son los más frecuentemente consultados y, por tanto, constituyen los temas de mayor interés para los estudiantes universitarios, quedando los últimos lugares reservados a aquellos que menos interés despiertan como son el horóscopo, la sociedad o crónica rosa, alimentación y moda.

Los hombres son lectores frecuentes y se interesan por los siguientes temas, en este orden: política, música, economía, deportes y cine, mientras que las mujeres tienen preferencia sobre todo por la música, el cine y la política.

Al analizar las diferencias por sexo entre los temas leídos al menos una vez por semana (Tabla VII), los hombres tienen más interés por los temas de política, economía, deportes y humor, mientras que los temas de salud, sociedad y crónica rosa, dieta, moda y horóscopo son más leídos por las mujeres.

\begin{tabular}{|l|r|r|r|r|r|}
\hline \multirow{2}{*}{} & \multicolumn{2}{c|}{ Hombres } & \multicolumn{2}{c|}{ Mujeres } & \multicolumn{1}{c|}{ p } \\
\cline { 2 - 5 } & $\mathrm{N}$ & \multicolumn{1}{c|}{$\%$} & $\mathrm{~N}$ & \multicolumn{1}{c|}{$\%$} & \\
\hline Educación & 105 & $46,9 \%$ & 156 & $46,3 \%$ & 0,892 \\
\hline Política & 163 & $72,8 \%$ & 173 & $51,3 \%$ & $<0,001$ \\
\hline Economía & 132 & $58,9 \%$ & 127 & $37,7 \%$ & $<0,001$ \\
\hline Salud & 51 & $22,8 \%$ & 102 & $30,3 \%$ & 0,051 \\
\hline Medio ambiente/naturaleza & 71 & $31,7 \%$ & 83 & $24,6 \%$ & 0,066 \\
\hline Consumo & 46 & $20,5 \%$ & 71 & $21,1 \%$ & 0,879 \\
\hline Deportes & 131 & $58,5 \%$ & 104 & $30,9 \%$ & $<0,001$ \\
\hline Sociedad/crónica rosa & 24 & $10,7 \%$ & 83 & $24,6 \%$ & $<0,001$ \\
\hline Alimentación/dieta & 23 & $10,3 \%$ & 64 & $19,0 \%$ & 0,005 \\
\hline Empleo & 68 & $30,4 \%$ & 116 & $34,4 \%$ & 0,315 \\
\hline Moda & 26 & $11,6 \%$ & 120 & $35,6 \%$ & $<0,001$ \\
\hline Música & 153 & $68,3 \%$ & 230 & $68,2 \%$ & 0,989 \\
\hline Cine & 127 & $56,7 \%$ & 212 & $62,9 \%$ & 0,141 \\
\hline Meteorología & 98 & $43,8 \%$ & 163 & $48,4 \%$ & 0,283 \\
\hline Horóscopo & 9 & $4,0 \%$ & 41 & $12,2 \%$ & 0,001 \\
\hline Humor: chistes, cómics & 101 & $45,1 \%$ & 102 & $30,3 \%$ & $<0,001$ \\
\hline
\end{tabular}

Tabla VII. Comparación de los temas leídos con frecuencia semanal entre hombres y mujeres. 
Lugares habituales de lectura: Las localizaciones más habituales de lectura son el propio domicilio (97,1\%), la biblioteca (71,5\%) y las aulas (66,5\%), mientras que los menos habituales son el bar o cafetería (41,5\%) y el lugar de trabajo (42,2\%) además de otros lugares inespecíficos (34,9\%). Al analizar los lugares de lectura en función de días laborales o fines de semana, se observa que en los días laborales siguen predominando como emplazamientos el domicilio, la biblioteca y el aula, mientras que durante los fines de semana, además del domicilio, se utilizan más los espacios públicos y sociales como parques o plazas y bares o cafeterías.

\begin{tabular}{|l|c|c|c|c|c|c|}
\hline & \multicolumn{2}{|c|}{ Laborables } & \multicolumn{2}{c|}{ Fines de semana } & \multicolumn{2}{c|}{ Total } \\
\hline & $\mathrm{N}$ & \multicolumn{1}{c|}{$\%$} & $\mathrm{~N}$ & \multicolumn{1}{c|}{$\%$} & $\mathrm{~N}$ & $\%$ \\
\hline Domicilio & 486 & $86,6 \%$ & 438 & $78,1 \%$ & 545 & $97,1 \%$ \\
\hline Espacios públicos (parques, plazas...) & 139 & $24,8 \%$ & 174 & $31,0 \%$ & 258 & $46,0 \%$ \\
\hline Transporte público & 278 & $49,6 \%$ & 96 & $17,1 \%$ & 300 & $53,5 \%$ \\
\hline Lugar de trabajo & 227 & $40,5 \%$ & 30 & $5,3 \%$ & 237 & $42,2 \%$ \\
\hline Bar / cafetería & 167 & $29,8 \%$ & 124 & $22,1 \%$ & 233 & $41,5 \%$ \\
\hline Biblioteca & 378 & $67,4 \%$ & 110 & $19,6 \%$ & 401 & $71,5 \%$ \\
\hline Aula & 367 & $65,4 \%$ & 45 & $8,0 \%$ & 373 & $66,5 \%$ \\
\hline Otros lugares & 115 & $20,5 \%$ & 155 & $27,6 \%$ & 196 & $34,9 \%$ \\
\hline
\end{tabular}

Tabla VIII. Lugares habituales de lectura.

Formato de información preferible para su lectura: Ante la pregunta de qué formato de información prefieren leer los estudiantes, en casi todos los casos se inclinan por la información impresa, siendo estas preferencias mucho más marcadas cuando se trata de literatura, libros académicos y cómics, y algo menos si se refiere a prensa y revistas. Únicamente muestran preferencia por el formato digital cuando se trata de artículos científicos, probablemente debido a que este tipo de información es accesible inicialmente en digital y están acostumbrados a leerlos en la pantalla del ordenador en lugar de imprimirlos.

Hay que destacar que los estudiantes, a pesar de que leen más en formato digital la información de revistas, libros de texto y literatura, prefieren el soporte tradicional que representa el papel frente al formato digital utilizado en los dispositivos electrónicos (ordenadores, e-books, tabletas y smartphones).

\begin{tabular}{|l|c|c|c|c|c|}
\hline & \multicolumn{2}{|c|}{ Papel } & \multicolumn{2}{c|}{ Digital } & Total \\
\hline Literatura & N & $\%$ & N & $\%$ & N \\
\hline Libros académicos & 461 & $87,3 \%$ & 67 & $12,7 \%$ & 528 \\
\hline Artículos científicos & 442 & $83,1 \%$ & 90 & $16,9 \%$ & 532 \\
\hline Prensa, revistas & 222 & $43,9 \%$ & 284 & $56,1 \%$ & 506 \\
\hline Cómics & 312 & $58,9 \%$ & 218 & $41,1 \%$ & 530 \\
\hline
\end{tabular}

Tabla IX. Preferencia de lectura de información en soporte impreso o digital.

\section{DISCUSIÓN}

Los resultados obtenidos en cuanto al tipo y formato de información utilizada de manera habitual por los estudiantes universitarios destacan porque muchos de ellos (entre el 92\% y el 98\%) consumen información de páginas web y utilizan el correo electrónico, WhatsApps, foros, o chats como medios de comunicación, además de ver películas y oír música a través de internet. Las redes sociales son utilizadas en menor medida, pero también por un porcentaje muy alto (88,6\%) que desciende hasta el 79,7\% de uso diario o casi diario, cifra algo más baja que la obtenida en el estudio de Iglesias García y González Díaz (2012) en el que el 89,9\% de los estudiantes de primero de Grado en Publicidad y Relaciones Públicas de la Universidad de Alicante las usaban todos los días.

El 64,3\% de estudiantes universitarios leen prensa digital y/o en papel una vez por semana, porcentaje algo inferior al obtenido por Rodríguez Legido (2009) en estudiantes de universidades de Andalucía (70\%). Este porcentaje baja a casi la mitad (46,6\%) cuando se trata de lectura de prensa digital todos o casi todos los días, porcentaje que aun así es muy superior al obtenido por Iglesias García y González Díaz (2012), donde sólo el 15\% leía prensa digital. En este mismo estudio los estudiantes apenas leían prensa en papel porque solo tratan temas de política, lo que discrepa notablemente con nuestro estudio ya que la política es uno de los temas que más interesa y el 34\% leen todos o casi 
todos los días cuestiones relacionadas con la política, siendo el tema más leído por los hombres (72,8\% al menos una vez por semana).

La lectura de libros de texto o material docente es un hábito realizado por la mayoría de los estudiantes, mucho más cuando se trata de libros de texto en papel (90,7\% de los estudiantes) que cuando se trata del formato digital $(55,4 \%)$, lo que puede deberse a que en las asignaturas de los grados de la Facultad de Filosofía y Letras todavía se sigue recomendando y utilizando mucha bibliografía impresa. Estos porcentajes son superiores a los obtenidos en un estudio realizado en estudiantes universitarios de últimos cursos de medicina (Al Husaini, 2013), en el que el 47,6\% leían libros de texto de todo tipo y solo un 39\% leían libros de texto online.

La fuente de información menos utilizada corresponde a las revistas, aunque se leen casi tres veces más en papel que en formato digital. Al distinguir las revistas de ocio de las revistas académicas, se observa que se leen más las primeras en formato digital que las segundas (17,6\% semanalmente frente al 11,8\%, respectivamente). Este porcentaje es sensiblemente inferior al de los estudiantes de medicina que leen artículos de revistas online (27,6\%) en el estudio de Al Husaini (2013). La explicación de este bajo porcentaje de estudiantes que leen artículos de revistas académicas online puede deberse a que no es un recurso habitual en la bibliografía de muchas asignaturas de Humanidades, en las que predominan las monografías.

En la Tabla X se comparan los resultados obtenidos en nuestro estudio con los conseguidos por diversos autores en estudiantes universitarios (Shen, 2012; Abdul Karim y Hasan, 2007; Noorizah, 2011; Federación de Gremios de Editores de España, 2013). Destaca que las preferencias de lectura de los universitarios es la información online o páginas web, siendo el correo electrónico el medio y la fuente de información que ocupa el segundo lugar. También hay coincidencia en que el porcentaje más bajo corresponde a los artículos de revistas académicas (entre el 11,3\% y el $12 \%$ en los tres estudios), una cifra que se considera muy baja teniendo en cuenta que las universidades tienen suscripciones con proveedores de información a texto completo y el aumento cada vez mayor del número de artículos a texto completo disponibles de forma gratuita en internet.

\begin{tabular}{|l|l|l|l|l|l|}
\hline & $\begin{array}{c}\text { Shen } \\
(\mathbf{2 0 1 2})\end{array}$ & $\begin{array}{c}\text { Karim } \\
\mathbf{( 2 0 0 7 )}\end{array}$ & $\begin{array}{c}\text { Noorizah } \\
\mathbf{( 2 0 1 1 )}\end{array}$ & $\begin{array}{c}\text { FGEE } \\
\mathbf{( 2 0 1 2})\end{array}$ & $\begin{array}{c}\text { Estudio } \\
\text { propio }\end{array}$ \\
\hline Información online (páginas web) & $83,9 \%$ & $70 \%$ & $88 \%$ & $65,4 \%$ & $98,2 \%$ \\
\hline Correo electrónico & $69,3 \%$ & & $85 \%$ & & $96,6 \%$ \\
\hline Prensa & $31,4 \%$ & $74 \%$ & & $59,2 \%$ & $64,3 \%$ \\
\hline Revistas digitales & $33,1 \%$ & & & $12,9 \%$ & $23,7 \%$ \\
\hline Revistas en papel & & & $67 \%$ & & $64,2 \%$ \\
\hline Artículos de revistas & $11,3 \%$ & $12 \%$ & & & $11,8 \%$ \\
\hline Libros de texto en papel & & $72 \%$ & $81 \%$ & & $90,7 \%$ \\
\hline Libros de texto online & $32,3 \%$ & & & & $55,4 \%$ \\
\hline Literatura digital (libros digitales) & $19,3 \%$ & $11 \%$ & & $18,5 \%$ & $32,3 \%$ \\
\hline Literatura (libros impresos) & & & $69 \%$ & $72,2 \%$ & $88,1 \%$ \\
\hline
\end{tabular}

Tabla X. Comparación de los resultados en lectura con otros estudios.

Existe cierta disparidad en los resultados de la lectura de literatura tanto impresa como digital, obteniendo en nuestro estudio los porcentajes más altos, lo que puede ser debido a que se ha llevado a cabo en una facultad de humanidades y en diversos grados se exige a los alumnos la lectura de este tipo de obras.

Es importante destacar que a pesar de la popularización de los libros electrónicos, predomina la lectura de los libros en papel frente a los libros digitales. Así consta también en un estudio realizado en Estados Unidos donde se pone de manifiesto que el libro impreso sigue siendo la base de los hábitos de lectura de los americanos, con un $69 \%$ de los adultos (18 o más años) que leen un libro impreso frente al 28\% que lo hacen en formato digital (Pew Research Center, 2014). La preferencia de la lectura de los libros impresos frente al formato electrónico se debe principalmente a dos razones, su fácil lectura y su portabilidad (Camacho y Spackman, 2011).

Las preferencias de lectura de información en papel frente al formato digital se ponen de manifiesto en nuestro estudio, con diferencias que oscilan entre porcentajes superiores al 80\% para literatura y libros académicos en papel a porcentajes inferiores al $17 \%$ en formato digital. Estos resultados están en consonancia con los que aparecen en la literatura científica, y así, en una revisión de diversos estudios, en todos ellos los estudiantes prefieren leer en papel

* Federación de Gremios de Editores de España (datos de estudiantes universitarios). 
(Cull, 2011); en el artículo de Vandenhoek (2013) el 74 \% de los universitarios escogen leer en papel, así como en el de Cornejo et al. (2012) en el que el 91,1\% de los alumnos universitarios de Ciencia y Tecnología leen libros en papel frente a un $27,2 \%$ que lo hace en formato electrónico.

Entre las razones apuntadas se aduce que a los usuarios les desagrada la lectura en pantalla durante largos periodos de tiempo (Ackerman y Goldsmith, 2011) o leer textos extensos en pantallas de ordenador (Gunter, 2005), y que el papel impreso es el mejor vehículo para una lectura cómoda y agradable durante un periodo largo de tiempo.

Es preciso destacar que existen diferencias significativas entre los hábitos de lectura de hombres y mujeres según las temáticas. Aunque la política interesa a ambos sexos, los hombres se interesan más que las mujeres y casi las tres cuartas partes son lectores habituales. También son de más interés para los hombres los temas relacionados con la economía, deportes y humor, contenidos propios de prensa y cómics; por el contrario, las mujeres se inclinan más por noticias de sociedad, moda y horóscopo, contenidos publicados en revistas. Estos resultados concuerdan con los obtenidos por la Federación de Gremios de Editores de España (2013), donde se indica que las mujeres leen más libros y revistas mientras que los hombres leen más prensa, cómics, blogs y foros.

Respecto al lugar más frecuente de lectura, la mayoría de los estudiantes universitarios declara que lo hace en su domicilio, y así se pone de manifiesto también en otros estudios (Cornejo et al., 2012), con porcentajes muy similares y superiores al 90\%. Otros lugares habituales de lectura son las bibliotecas y el aula, espacios habituales de trabajo y estudio. Así mismo, más de la mitad de los encuestados manifiestan que leen en el transporte público.

\section{CONCLUSIONES}

Los resultados generales de este estudio revelan que los estudiantes universitarios de la Facultad de Filosofía y Letras de la Universidad de Zaragoza son lectores y consumidores frecuentes de una amplia variedad y tipos de información. Entre estas destacan las páginas web. Por el contrario, las revistas, tanto de ocio como académicas, son las fuentes de información menos leídas.

Utilizan múltiples dispositivos, prácticamente todos los estudiantes usan el ordenador y las tres cuartas partes el smartphone. El uso de tabletas y lectores de libros electrónicos está poco extendido, en el primer caso para acceder a información de ocio y como dispositivo de comunicación, y en el segundo caso para leer literatura y libros de texto.

Más de la mitad de los universitarios son lectores y consumidores frecuentes de temas relacionados con la música, cine y política, siendo los relacionados con el horóscopo, sociedad, crónica rosa, dieta y moda los que despiertan menos interés, si bien es un tipo de información consumida más por las mujeres, inclinándose los hombres más por la política, economía y deportes.

Los lugares habituales de lectura son el domicilio y la biblioteca, aunque en los fines de semana los espacios públicos como parques ocupan el segundo lugar.

En cuanto a los tipos de información que coexisten en soporte digital e impreso, los universitarios prefieren leer en papel, sobre todo literatura, libros y cómics.

Hay un porcentaje importante de estudiantes que leen material docente digital, consecuencia de la aparición y mayor uso de plataformas digitales utilizadas en el proceso de enseñanza-aprendizaje en el ámbito universitario. El uso de manuales, libros de texto, revistas y diferentes fuentes de información aumenta la práctica de la lectura y, por tanto, produce un aumento del hábito lector. Serán necesarios otros estudios para averiguar si estos hábitos son eficaces en cuanto a la adquisición de nuevo conocimiento y suponen una mejora en el rendimiento académico o bien la lectura se hace de manera superficial y principalmente dirigida a actividades de ocio.

La lectura está íntimamente ligada al aprendizaje, por lo que es necesario promover hábitos de lectura en los estudiantes universitarios y motivarlos para que lean diferentes tipos de información y utilicen diferentes canales o medios (revistas, libros de texto, materiales docentes, etc.) y conseguir así un buen rendimiento académico y enriquecimiento cultural personal. En la lectura se emplean diferentes estrategias según sea el objetivo a alcanzar, por placer, para aprender o simplemente para informarse. Hay que pensar en la lectura también como actividad de ocio y entretenimiento, no solo como tarea necesaria superar las asignaturas. Con los nuevos dispositivos y formas de lectura es importante encontrar la manera de estimular el interés por la lectura y de mejorar los hábitos lectores. 
Son necesarios más estudios para identificar y comprender los problemas asociados con los hábitos de lectura en todos los niveles educativos, desde la escuela primaria a la universidad, e implementar soluciones eficaces que faciliten a los estudiantes la adquisición de nuevo conocimiento.

\section{BIBLIOGRAFÍA}

ABDUL KARIM, N.S. y HASAN, A. Reading habits and attitude in the digital age. Analysis of gender and academic program differences in Malasya. The Electronic Library, 2007, vol. 2, $\mathrm{n}^{\circ}$ 3, p. 285-298. Doi: http://dx.doi.org/10.1108/02640470710754805.

ACKERMAN, R. y GOLDSMITH, M. Metacognitive regulation of text learning: On screen versus on paper. Journal of Experimental Psychology: Applied, 2011, vol. 17, p. 18-32. Doi: http://dx.doi.org/10.1037/a0022086.

AKANDA, A.K.M.; HOQ, K.M.G. y HASAN, N. Reading habit of students in social sciences and arts: A case study of Rajshahi University. Chinese Librarianship: an International Electronic Journal, 2013, vol. 35. $<$ http://www.iclc.us/cliej/cl35AHH.pdf>. [Consulta: 24 de abril de 2014].

AL HUSAINI, Z.A.E. Knowledge, attitude and practice of reading habit among female medical students, Taibah University. Journal of Taibah University Medical Sciences, 2013, vol. 8, nº 3, p. 192-198.

ALONSO ARÉVALO, J.; CORDÓN GARCÍA, J.A. y GÓMEZ DÍAZ, R. Comparación de los hábitos y perfil del lector digital entre Estados Unidos y España. Anales de Documentación, 2014, vol. 17, $\mathrm{n}^{\mathrm{o}} 1$. Doi: $^{\circ}$ http://dx.doi.org/10.6018/analesdoc.17.1.193111.

BROWN, G.J. Beyond print: Reading digitally. Library Hi Tech, 2001, vol. 19, nº 4, p. 390-399.

CAMACHO, L. y SPACKMAN, A. Transitioning to E-books: Usage and attitudes among Business Faculty. Journal of Business and Finance Librarianship, 2011, vol. 16, p. 33-45.

CORNEJO, J.N.; ROBLE, M.B.; BARRERO, C. y MARTÍN, A.M. Hábitos de lectura en alumnos universitarios de carreras de Ciencia y Tecnología. Revista Eureka sobre Enseñanza y Divulgación de las Ciencias, 2012, vol. 9, $\mathrm{n}^{\circ}$ 1, p. 155-163.

CULL, B. Reading revolution: Online digital texts and implications for reading in academics. First Monday Peer reviewed Journal on the Internet, 2011, vol. $16, \quad \mathrm{n}^{\circ} \quad 6$. $<$ http://firstmonday.org/ojs/index.php/fm/article/view/3340/2985>. [Consulta: 9 de abril de 2014].

CUMAOLGU, G.; SACICI, E. y TORUM, K. E-book versus printed materials: preferences of university students. Contemporary Educational Technology, 2013, vol. 4, n² 2, p. 121-135.

DÍAZ GÓMEZ, J.M. y GÁMEZ ARMAS, E. Hábitos lectores y motivación entre estudiantes universitarios. REME Revista Electrónica de Motivación y Emoción, 2003, vol. 6, $n^{\circ} 13$. $<$ http://reme.uji.es/articulos/adxazj7690710102/texto.html>. [Consulta: 25 de abril de 2014].

Federación de Gremios de Editores de España. Hábitos de lectura y compra de libros en España 2012. Madrid: Federación de Gremios de Editores de 2013. <http://www.mcu.es/libro/docs/MC/Observatorio/pdf/Habitos_lectura_2012.pdf>. [Consulta: 24 de abril de 2014].

GARCÍA DE BLAS, E. El futuro examen digital de PISA amenaza con rebajar más las notas. El País, 6 de junio de 2014, p. 36-37.

GARCÍA DELGADO, B. Estudio de los hábitos lectores de los estudiantes de la Universidad Europea de Madrid. Ibersid, 2011, vol. 5, p. 99-107.

GIL FLORES, J. Hábitos lectores y competencias básicas en el alumnado de Educación Secundaria Obligatoria. Educación XX1, 2011, vol. 14, nº 1. Doi: http://dx.doi.org/10.5944/educxx1.14.1.274.

GUNTER, B. Electronic books: A survey of users in the UK. Aslib Proceedings: New Information Perspectives, 2005, vol. 57, p. 513-522.

IGLESIAS GARCÍA, M. y GONZÁLEZ DÍAZ, C. Radiografía del consumo de medios de comunicación en estudiantes universitarios. Icono 14, 2012, vol. 10, nº 3, p. 100-115. Doi: http://dx.doi.org/10.7195/ri14.v10i3.212.

KELLER, A. In print or on screen? Investigating the reading habits of undergraduate students using pho-diaries and photo-interviews. Libri, 2012, vol. 62, p. 1-18. Doi: http://dx.doi.org/10.1515/libri-2012-0001.

LARRAÑAGA RUBIO, E. La lectura en los estudiantes universitarios: variables psicosociales en la formación de los hábitos lectores. [Tesis doctoral]. Cuenca: Universidad de Castilla-La Mancha, 2004.

LARRAÑAGA, E. y MARTÍNEZ, I. Un estudio sobre valores y hábitos lectores en jóvenes universitarios. En: Cerrillo, P.C.; Yubero, S.; Larrañaga, E. (coords.) Valores y lectura: estudios multidisciplinares. Cuenca: Ediciones de la Universidad de Castilla-La Mancha, 2004, p. 65-88.

LARRAÑAGA, E.; YUBERO, S. y CERRILLO, P.C. Estudio sobre los hábitos lectores de los estudiantes españoles. Cuenca: CEPLI; Fundación SM, 2008.

Ministerio de Educación, Cultura y Deporte. Informe español. PISA 2012. Resolución de problemas de la vida real. Resultados de Matemáticas y lectura por ordenador, 2013 
<https://www.mecd.gob.es/dctm/inee/internacional/pisa2012-resolucionproblemas/pisa2012cba-1-4-2014web.pdf?documentId=0901e72b8190478c > . [Consulta: 18 de abril de 2014].

NOORIZAH, M.N. Reading habits and preferences of EFL post graduates: A case study. Indonesian Journal of

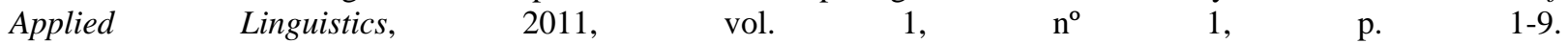
$<$ http://jurnal.upi.edu/file/01_Noorizah_Reading_habit_edited1.pdf>. [Consulta: 14 de abril de 2014].

ÖGEYIK M.C. y AKYAY, E. Investigating reading habits and preferences of student teachers at foreign language departments. The International Journal of Language Society and Culture, 2009, vol. 28, p. 72-78. <http://www.educ.utas.edu.au/users/tle/JOURNAL/issues/2009/28-7.pdf>. [Consulta: 21 de abril de 2014].

OLSEN, A.N.; KLEIVSET, B. y LANGSET, H. E-book readers in higher education. Student reading preferences and other data from surveys at the University of Adger. SAGE Open, 2013, vol. 3, n ${ }^{\circ} 2$. Doi: $^{2}$ http://dx.doi.org/10.1177/2158244013486493.

Pew Research Center. E-reading rises as device ownership jumps, 2014. <http://pewinternet.org/Reports/2014/EReading-Update.aspx>. [Consulta: 20 de abril de 2014].

PRENSKY, M. Digital Natives, Digital Immigrants. On the Horizon, 2001, vol. $9, \mathrm{n}^{\mathrm{o}} 5$. <http://www.marcprensky.com/writing/Prensky\%20-\%20Digital\%20Natives,\%20Digital\%20Immigrants\%20\%20Part1.pdf $>$. [Consulta: 21 de abril de 2014].

QUADRI, G.O. y ABOMOGE, S.O. A survey of reading and Internet use habits among undergraduate students in selected university libraries in Nigeria. Information and Knowledge Management, 2013, vol. 13, n ${ }^{\circ}$ 11, p. 38-46.

RODRÍGUEZ LEGIDO, C. Usos y hábitos de lectura en torno a la Universidad en Andalucía. Periférica: Revista para el análisis de la cultura y el territorio, 2009, vol. 10, p. 125-134.

SANTOS DÍEZ, M.T. y PÉREZ DASILVA, J.A. Diarios gratuitos de información general: percepción, hábitos de consumo y preferencias de lectura de los universitarios vascos. Estudios sobre el Mensaje Periodístico, 2010 , vol. 16, p. 437-455.

SCHUGAR, J.T.; SCHUGAR, H. y PENNY, C. A nook or a book? Comparing college student's reading comprehension levels, critical reading and study skills. International Journal of Technology in Teaching and Learning, 2011, vol. 7, p. 174-192. <http://www.sicet.org/journals/ijttl/issue1102/6_Schugar.pdf>. [Consulta: 22 de abril de 2014].

SHEN, L.B. Computer technology and college student's reading habits. Chia-Nan Annual Bulletin, 2006, vol. 32, p. 559-572. <http://lib.chna.edu.tw/bulletin/files/v32_559_572.pdf>. [Consulta: 24 de abril de 2014].

SZAPKIW, A.J.R.; COURDUF, J.C.; CARTER, K. y BENNETT, D. Electronic versus traditional print textbooks: A comparison study on the influence of university students' learning. Computers \& Education, 2013, vol. 63, p. 259266.

TEBEROSKY, A.; GUARDIA, J. y ESCORIZA, J. Las prácticas de lectura en estudiantes universitarios. Anuario de Psicología, 1996, vol. 70, p. 85-107.

THANUSKODI, S. Reading habits among library and information science among students of Annamalai University: A survey. International Journal of Educational Sciences, 2011, vol. 3, n 2, p. 79-83.

VANDENHOEK, T. Screen Reading habits among university students. International Journal of Education and Development using Information and Communication Technology, 2013, vol. 9, $n^{\circ}$ 2, p. 37-47.

YUBERO, S. y LARRAÑAGA, E. El valor de la lectura en relación con el comportamiento lector. Un estudio sobre los hábitos lectores y el estilo de vida en niños. OCNOS, 2010, vol. 6, p. 7-20.

YUBERO, S.; LARRAÑAGA, E. y CERRILLO, P.C. El valor de la lectura en la formación del hábito lector. En: Martos, E. y Rösing T.M.K. (coords.). Prácticas de lectura y escritura. Passo Fundo: Editora da Universidade de Passo Fundo, 2009, p. 115-136. 
Anexo 1. Cuestionario de hábitos de lectura

$\begin{array}{ll}\text { 1. Sexo: } \square \text { 2. Mujer } & \text { 2. Edad: } \square \\ \text { 3. Titulación: } & \end{array}$

4. Curso en el que está matriculado:

5. Indique si utiliza, al menos una vez por semana, los dispositivos o soportes señalados para los siguientes formatos de información:

0.- No

1.- Sí

9.- No tiene dispositivo o no procede

\begin{tabular}{|c|c|c|c|c|c|c|}
\hline & En papel & $\begin{array}{l}\text { Ordenador } \\
\text { sobremesa }\end{array}$ & $\begin{array}{l}\text { Ordenador } \\
\text { portátil }\end{array}$ & $\begin{array}{l}\text { Smart- } \\
\text { phone }\end{array}$ & Tableta & e-book \\
\hline \multicolumn{7}{|l|}{ Prensa } \\
\hline \multicolumn{7}{|l|}{ Revistas } \\
\hline \multicolumn{7}{|c|}{ Libros de texto/mat.docente } \\
\hline \multicolumn{7}{|l|}{ Literatura } \\
\hline \multicolumn{7}{|l|}{ Páginas web } \\
\hline \multicolumn{7}{|c|}{ Correo electrónico/SMS } \\
\hline \multicolumn{7}{|c|}{ Blogs, foros, chats, WhatsApps } \\
\hline \multicolumn{7}{|l|}{ Redes sociales } \\
\hline \multicolumn{7}{|c|}{ Películas, vídeos } \\
\hline Música, audio & & & & & & \\
\hline
\end{tabular}

6. Indique con qué frecuencia lee en Internet los diferentes tipos de información que se indican: Por favor, rodee con un círculo su respuesta.

\begin{tabular}{|c|c|c|c|c|c|c|}
\hline & Nunca & $\begin{array}{c}\text { Casi } \\
\text { nunca }\end{array}$ & $\begin{array}{c}\text { Al menos, } \\
\text { una vez } \\
\text { cada tres } \\
\text { meses }\end{array}$ & $\begin{array}{c}\text { Al menos, } \\
\text { una vez al } \\
\text { mes }\end{array}$ & $\begin{array}{c}\text { Una o dos } \\
\text { veces por } \\
\text { semana }\end{array}$ & $\begin{array}{l}\text { Todos o } \\
\text { casi todos } \\
\text { los días }\end{array}$ \\
\hline Revistas de ocio & 1 & 2 & 3 & 4 & 5 & 6 \\
\hline Revistas académicas, científicas & 1 & 2 & 3 & 4 & 5 & 6 \\
\hline Prensa & 1 & 2 & 3 & 4 & 5 & 6 \\
\hline Libros de texto/material docente & 1 & 2 & 3 & 4 & 5 & 6 \\
\hline Literatura & 1 & 2 & 3 & 4 & 5 & 6 \\
\hline Redes sociales & 1 & 2 & 3 & 4 & 5 & 6 \\
\hline Correo electrónico & 1 & 2 & 3 & 4 & 5 & 6 \\
\hline Blogs/foros/chats & 1 & 2 & 3 & 4 & 5 & 6 \\
\hline
\end{tabular}


7. Indique la frecuencia con que lee información en Internet sobre los siguientes temas: Por favor, rodee con un círculo su respuesta.

\begin{tabular}{llccccc}
\hline & Nunca & $\begin{array}{c}\text { Casi } \\
\text { nunca }\end{array}$ & $\begin{array}{c}\text { Al menos, } \\
\text { una vez } \\
\text { cada tres } \\
\text { meses }\end{array}$ & $\begin{array}{c}\text { Al menos, } \\
\text { una vez al } \\
\text { mes }\end{array}$ & $\begin{array}{c}\text { Una o dos } \\
\text { veces por } \\
\text { semana }\end{array}$ & $\begin{array}{c}\text { Todos o } \\
\text { casi todos } \\
\text { los días }\end{array}$ \\
\hline Educación & 1 & 2 & 3 & 4 & 5 & 6 \\
\hline Política & 1 & 2 & 3 & 4 & 5 & 6 \\
\hline Economía & 1 & 2 & 3 & 4 & 5 & 6 \\
\hline Salud & 1 & 2 & 3 & 4 & 5 & 6 \\
\hline Medio ambiente/naturaleza & 1 & 2 & 3 & 4 & 5 & 6 \\
\hline Consumo & 1 & 2 & 3 & 4 & 5 & 6 \\
\hline Deportes & 1 & 2 & 3 & 4 & 5 & 6 \\
\hline Sociedad/crónica rosa & 1 & 2 & 3 & 4 & 5 & 6 \\
\hline Alimentación/dieta & 1 & 2 & 3 & 4 & 5 & 6 \\
\hline Empleo & 1 & 2 & 3 & 4 & 5 & 6 \\
\hline Moda & 1 & 2 & 3 & 4 & 5 & 6 \\
\hline Música & 1 & 2 & 3 & 4 & 5 & 6 \\
\hline Cine & 1 & 2 & 3 & 4 & 5 & 6 \\
\hline Meteorología & 1 & 2 & 3 & 4 & 5 & 6 \\
\hline Horóscopo & 1 & 2 & 3 & 4 & 5 & 6 \\
\hline Humor: chistes, cómics & 1 & 2 & 3 & 4 & 5 & 6 \\
\hline
\end{tabular}

8. Marque con una cruz los lugares en los que suele leer habitualmente (al menos, una vez a la semana), bien sea en días laborales o en fines de semana/vacaciones

\begin{tabular}{|l|c|}
\hline & Dias laborales \\
\hline En su domicilio & $\begin{array}{c}\text { Fines de } \\
\text { semana/vacaciones }\end{array}$ \\
\hline En espacios públicos (parques, plazas.) & \\
\hline En el transporte público & \\
\hline En el lugar de trabajo & \\
\hline En un bar/cafetería & \\
\hline En una biblioteca & \\
\hline En el aula & \\
\hline En otros lugares:
\end{tabular}

\section{Marque con una cruz la preferencia en la lectura de los siguientes tipos de textos:}

\begin{tabular}{l}
\hline Literatura (narrativa, poesia, teatro...) \\
Libros académicos (textos relacionados con mi área de estudio o profesional) \\
Artículos científicos \\
Prensa, revistas \\
Cómics
\end{tabular}

